\title{
Overall design of JASMINE
}

\author{
Yoshiyuki Yamada ${ }^{1}$, Naoteru Gouda ${ }^{2}$, Takuji Tsujimoto ${ }^{2}$, \\ Yukiyasu Kobayashi ${ }^{2}$, Tadashi Nakajima ${ }^{2}$, Hideo Matsuhara ${ }^{3}$, \\ Taihei Yano ${ }^{2}$, Seiji Ueda ${ }^{4}$, Masahiro Suganuma ${ }^{2}$, \\ and the JASMINE Working Group \\ ${ }^{1}$ Department of Physics, Kyoto University, Kyoto, 606-8502, JAPAN \\ email: yamada@amesh.org \\ ${ }^{2}$ National Astronomical Observatory of JAPAN, National Institutes of Natural Sciences, \\ Mitaka, Tokyo, 181-8588, JAPAN \\ email:naoteru.gouda@nao.ac.jp,taku.tsujimoto@nao.ac.jp, \\ yuki@merope.mtk.nao.ac.jp,tadashi@dodgers.mtk.nao.ac.jp, \\ t.yano@nao.ac.jp,suganuma@merope.mtk.nao.ac.jp \\ ${ }^{3}$ Institute of Space and Astronautical Science, Japan Aerospace Exploration Agency(JAXA), \\ Sagamihara, Kanagawa, 229-8510, JAPAN \\ email:maruma@ir.isas.ac.jp \\ ${ }^{4}$ The Graduate University for Advanced Studies, Mitaka, Tokyo, 181-8588, JAPAN \\ email:seiji.ueda@nao.ac.jp
}

\begin{abstract}
JASMINE (Japan Astrometry Satellite Mission for INfrared Exploration) is a mission to determine positions and parallaxes accurate to $\sim 10 \mu$ arcsec, with proper motion errors $\sim 10 \mu \operatorname{arcsec} \mathrm{yr}^{-1}$ for Galactic stars observed in the z-band $(0.9 \mu \mathrm{m})$. In this article, we report some technical investigations.
\end{abstract}

\section{Introduction}

JASMINE (Japan Astrometry Satellite Mission for INfrared Exploration) is a mission to determine positions and parallaxes accurate to $\sim 10 \mu \operatorname{arcsec}$, with proper motion errors

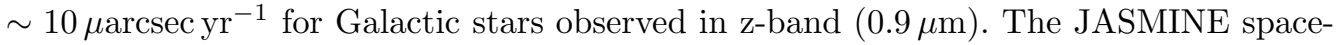
craft rotates slowly with a rotation axis aligned 3.5 from the Galactic pole-spacecraft line to perform a continuously scanning observation. This precession makes the JASMINE spacecraft scan around the Galactic plane with a $360^{\circ} \times 7^{\circ}$ area.

The JASMINE instrument uses a beam combiner to observe two fields of view separated by 99.5 simultaneously. This makes it possible to perform global measurements to get absolute parallaxes. The two fields of view are fed into a common telescope whose detailed design is described by Yano et al. (these proceedings). The present mission and instrument design are summarized in Table 1.

JASMINE will be launched around 2014 and a candidate for the orbit will be a Lissajous orbit around the Sun-Earth L2 point with a 5 -yr mission lifetime. The stability requirement for rotation of the satellite is about $6 \times 10^{-6} \mathrm{degs}^{-1}(t<1 \mathrm{~s}$ where $t$ is the sampling rate). The stability requirement of the relative angle of a beam combiner is less than $10 \mu$ as during the period of the spacecraft rotation. These requirements are very important technical issues for achievement of the required astrometric accuracies. Detailed consideration of the overall system design continues in cooperation with JAXA (Japan Aerospace Exploration Agency).

In Section 2 we show the method for calculating JASMINE specifications, and Section 3 contains discussions of several technical developments. In this paper, we limit ourselves to 
Table 1. Overview of system design

\begin{tabular}{ll}
\hline \multicolumn{2}{c}{ Mission Parameter } \\
\hline Mission Time & $5 \mathrm{yr}$ \\
Rotation Period & $\sim 5.0 \mathrm{hr}$ \\
Precission Period & $28.6 \mathrm{~d}$ \\
Rotation Axis & around the Galactic Pole \\
\hline \multicolumn{1}{c}{ Optical } & System Parameter \\
\hline Aperture size & $\sim 1.5 \mathrm{~m}$ \\
Focal length & $\sim 50.0 \mathrm{~m}$ \\
pixel size & $15 \mu \mathrm{m}$ \\
pixel on the sky & $\sim 61.9 \mathrm{mas}$ \\
Basic angle & 99.5 \\
\hline
\end{tabular}

technical items which other papers about JASMINE in these proceedings presentation do not cover. We are constructing integrated simulation software for the JASMINE mission design (JASMINE Simulator; see Ueda et al., these proceedings). Optical system design and centroiding algorithms are introduced by Yano et al. (these proceedings)and for JASMINE scientific objectives, see Gouda et al. (these proceedings).

\section{Specification}

Specifications for JASMINE are calculated as follows. From centroiding experiments (see Yano et al., these proceedings), the size of the point spread function in focal length is chosen as $N_{P S F}=2$ pixels.

$$
\frac{\lambda}{D} \frac{f}{w}=N_{P S F}=2
$$

where $\lambda, D, f$, and $w$ are wavelength, diameter of primary mirror, focal length of the optics, and pixel size, respectively. The angular sizes of the fields of view for one detector, $\alpha_{s}$ and $\alpha_{c}$, are

$$
\alpha_{s / c}=\frac{N_{p i x-s / c} w}{f}=\frac{N_{p i x-s / c}}{N_{P S F}} \frac{\lambda}{D}
$$

where indices $s$ and $c$ are scanned and cross-scanned direction, respectively.

The number of photons $N_{\mathrm{ph}}$ for achieving the required accuracy for JASMINE $(\sigma=$ $10 \mu \operatorname{arcsec} \mathrm{rms})$ is calculated by an equation

$$
\sigma=c \frac{\lambda}{D \sqrt{N_{\mathrm{ph}}}}=10 \mu \operatorname{arcsec}
$$

where $c$ is constant (assumed to be 0.5 ).

The number of photons observed per unit time $F_{m}$ is denoted as

$$
F_{m}=A \varepsilon E_{0} 10^{-0.4 m} / h,
$$

where $A, E_{0}$, and $h$ are the area of primary mirror, energy flux of 0 magnitude stars, and the Planck constant, respectively. The value $\varepsilon$ represents total efficiency factor and multiples of four factors: (1) The average detector efficiency is now 0.7 but will be 0.9 within a few years; (2) The efficiency of the optics system is estimated as $(0.97)^{8} \sim 0.8$; (3) The number ratio of photons within the window of a stellar image $(5 \times 9$ pixels $)$ and total photons in our optical system $R_{w}=0.63$; (4) The band resolution $\Delta \lambda / \lambda \sim 0.22$. 


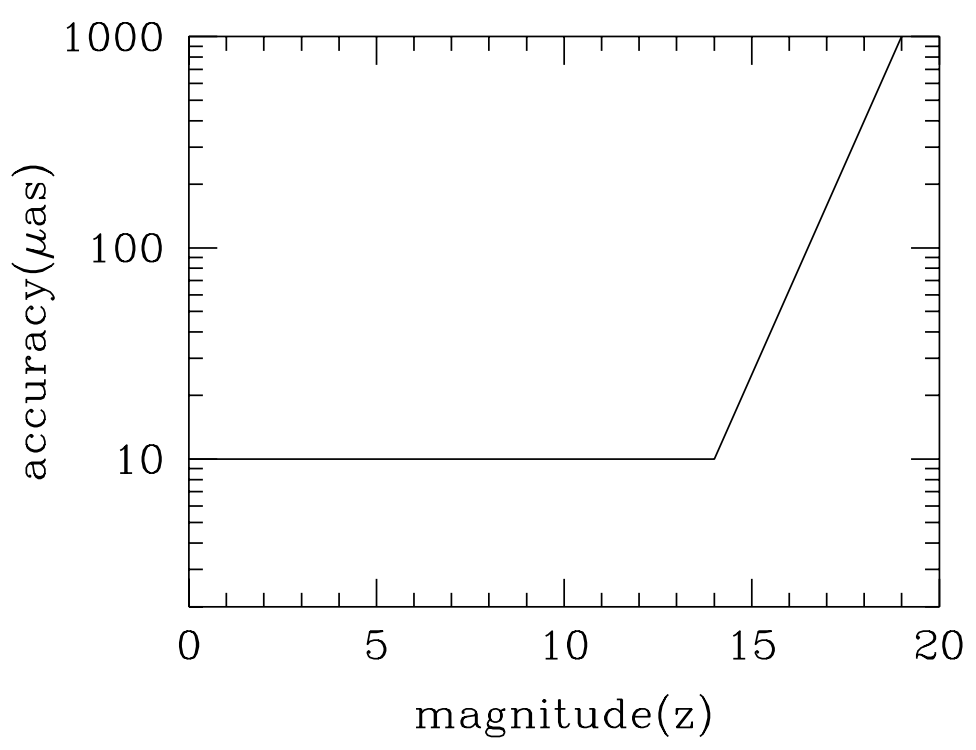

Figure 1. Position accuracy of JASMINE observations.

Using the above estimates, we get $\varepsilon \sim 0.1$. The effective area of our optical system is

$$
A=\frac{\pi}{4 N_{B C}}\left(D^{2}-D_{i n}^{2}\right)
$$

where $D \sim 1.5 \mathrm{~m}$ and $D_{i n} \sim 0.53 \mathrm{~m}$. The value $N_{B C}$ is the number of the beam combiner. The required integration time for determining the position of one $z=m(=14) \mathrm{mag}$ object with an accuracy of $10 \mu$ as is

$$
t_{i n t}=\frac{N_{p h}}{F_{m}}
$$

In our PSF, the ratio of the photons dropped into the central pixel and the total photons is $R_{c}=0.109$. We introduce a new constraint that an object of $z>m s(=9.8)$ does not saturate,

$$
R_{c} F_{m s} t_{i n t}=N_{e}
$$

where $N_{e}$ is full well capacity. For parameters of satellite motion, the periods of spin and precession are denoted as $T$ and $T_{\text {pole }}$, respectively.

$$
T=\frac{2 \pi}{\alpha_{s}} t
$$

and

$$
T_{\text {pole }}=\frac{2 \pi \sin i}{S \alpha_{c} n_{c}} T,
$$

where $i=3.5$ is the inclination of the precession axis, $n_{c}$ is the number of detectors along a cross scan, $S$ is ratio for the FOV change by precession. We introduce two other parameters, the duty-cycle of observation, $\beta$, and the mission time, $T_{m i s}$. The number of observations per year, $X$, is denoted as

$$
X=\beta \frac{1 \mathrm{yr}}{T_{\text {pole }}} N_{B C} n_{s} \frac{2}{S} .
$$




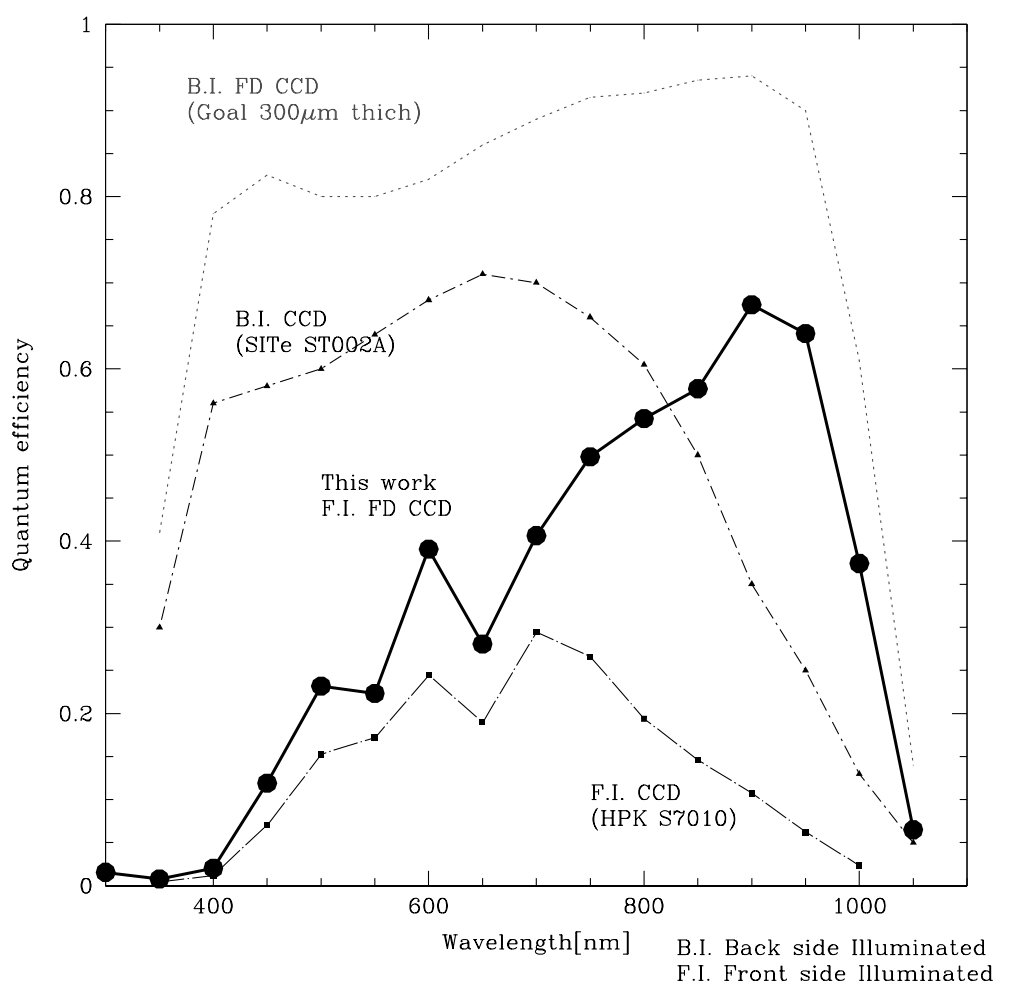

Figure 2. Quantum efficiency a prototype of the CCD under development.

On the other hand, the condition for the integration time is denoted as

$$
X T_{\text {mis }} t=t_{\text {int }} .
$$

Combining the above conditions, the total required number of detectors is

$$
\begin{aligned}
n_{c} n_{s} \sim & 88\left(\frac{c}{0.5}\right)^{2}\left(\frac{\sigma}{10 \mu \mathrm{as}}\right)^{-2}\left(\frac{A}{1.55 m^{2}}\right)^{-1}\left(\frac{\varepsilon}{0.1}\right)^{-1}\left(\frac{\beta}{0.4}\right)^{-1}\left(\frac{T_{m i s}}{5 \mathrm{yr}}\right)^{-1} \\
& \times\left(\frac{N_{p i x-s}}{4096}\right)^{-1}\left(\frac{N_{p i x-c}}{2048}\right)^{-1}\left(\frac{\sin i}{\sin 3.5 \mathrm{deg}}\right) .
\end{aligned}
$$

\section{Key Technologies}

The study objective is to identify technology activities that shall be performed before the Project Implementation Phase, aiming at reducing the project implementation costs. Some of studies are shown in other presentations in these proceedings: Optics design and centroidings (Yano et al., these proceedings); system simulation and software activities (Ueda et al., these proceedings).

\subsection{Detector}

On the focal plane, about $7 \times 14 \mathrm{CCDs}$ are assembled and they measure the stellar images in time-delayed integration (TDI) mode. We are developing a new type of CCD, a back-illuminated fully-depleted CCD for infrared astronomy. The quantum efficiency of a prototype of the CCD is shown in Fig 2 as a solid line. The efficiency at $0.9 \mu \mathrm{m}$ 


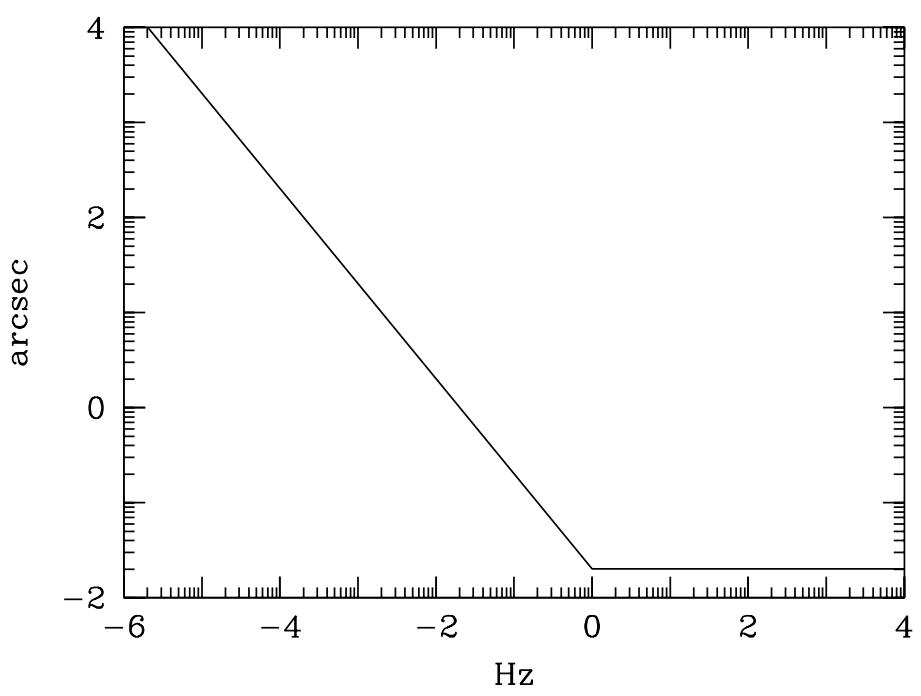

Figure 3. The requirement of satellite attitude control.

will be $90 \%$ in the near future. Impurity gathering techniques during the processing are employed to move impurities from the active sensor regions.

\subsection{Attitude requirement}

In our observations, the quality of the final scientific output is strongly dependent on the centroiding accuracy. In our simulation of centroiding algorithms, the satellite attitude is required to be controlled within 1 pixel for both scan and cross-scan directions. The requirement is shown in Fig. 3.

\subsection{On board data handling}

On-board object detection will ensure that variable stars, supernovae, transient sources, micro-lensed events, and minor planets will all be detected and catalogued to a faint limit. Each astrometric field comprises an astrometric sky mapper (ASM) and the astrometric field (AF). The sky mapper system provides an on-board capability for star detection and selection and for the star position and satellite scan-speed measurement. From the shape of the point spread function, timing of TDI operation is synchronized with satellite spin, and the spin axis is aligned with the detector implementation.

For each detected star, a window is selected around the star to optimise the complex trade-off between minimising read-out noise, maximising scientific return, and demanding feasible communications.

\subsection{Monitoring the basic angle}

JASMINE will measure the speed and position of a billion stars with extremely high precision (10 $\mu$ as accuracy). The lines-of-sight of the two telescopes are separated by an angle (called the "basic angle") of about 99:5. The basic angle stability should be within $10 \mu$ as rms over the satellite revolution period of $5 \mathrm{hr}$, or should be at least monitored with this accuracy. 


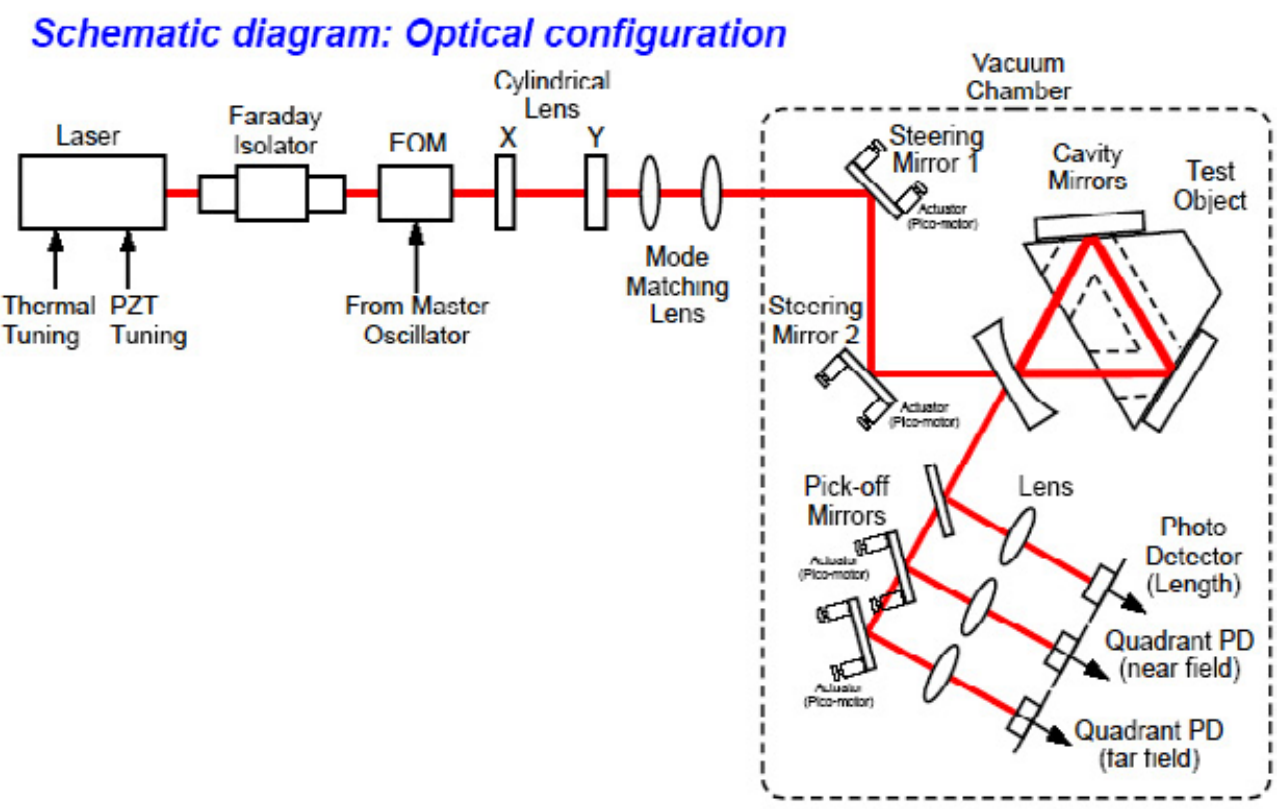

Figure 4. The basic angle monitor.

A technique of monitoring angle is developing in gravitational wave detection projects. The optimum localisation accuracy of the fringe pattern equals:

$$
\alpha=\frac{\lambda}{2 \pi B \sqrt{N}}
$$

where $\lambda, B, N$, and $\alpha$ are the wavelength, the length of the baseline, total number of detected photons, and the accuracy, respectively.

We have started ground-based experiments of basic angle monitor. The design of the instruments are shown in Fig. 4.

\subsection{Telecommunication}

The observed number of stars $(z<17 \mathrm{mag})$ is estimated to be $3.2 \times 10^{4}$ per square degree. If we use all data within the stellar window, $5 \times 9 \times 16$ bits are needed for each star. Considering the JASMINE specification parameters, 9.1 Mbps are required for data transfer. We assumed that telecommunication is enabled during $8 \mathrm{hr} /$ day. For transferring such large amount of data, a big parabolic antenna is needed, which may affect the attitude stability.

For telemetry data for faint stars, pixels in the cross-scan direction are integrated and a $5 \times 20$-bit stream is used. The advantage of this procedure is not only that it reduces data rates, but also decreases read-out noise. By doing this, data rates are reduced to $1.3 \mathrm{Mbps}$. Applying an appropriate loss-less data compression method, a phased array antenna can be applied for our telecommunication system. For data compression, Karhunen Loeve transformation and Rice compression may be applied.

\section{Conclusions}

JASMINE is a unique space astrometric mission using the infrared band. JASMINE will deliver huge scientific impact across the whole of astrophysics, especially the structure 
and evolution of the Galaxy. But JASMINE will observe only the Galactic disk and bulge. Maintaining close contact between the various proposed space astrometry missions is strongly required for understanding the whole of the Galactic structure. Since FY 2003, we have started collaboration with NASDA, ISAS (both institutes are unified to JAXA), Tokyo University, and the Gravitational wave detection team in NAOJ. System investigation has progressed very much. Until 2009, a mission proposal will be performed.

\section{Acknowledgements}

We would like to acknowledge Y. Kawakatsu, A. Noda, A. Tsuiki, M. Utashima, A. Ogawa, N. Sakou, H. Ueda for their collaboration in the investigations on the JASMINE spacecraft system. Furthermore we would like to tank S. Kawamura and K. Arai, who are members of TAMA-300 group. This work has been supported in part by the Grant-in-Aid for the Scientific Research Funds (15340066) and Toray Science Foundation. 